\title{
Fachliche Expertise und abweichendes Verhalten bei Abstimmungen zur Euro-Krise im Deutschen Bundestag
}

\author{
Andreas Wimmel
}

\begin{abstract}
Kernaussagen
Im Zuge der Euro-Krise haben sich in fast allen Bundestagsfraktionen kleine Gruppen von Abgeordneten formiert, die die von der Bundesregierung auf europäischer Ebene vereinbarten Euro-Rettungsmaßnahmen nicht unterstützen. Wie lässt sich dieses abweichende Verhalten bei namentlichen Abstimmungen erklären? Ein Vergleich mit der Mehrheit der zustimmenden Abgeordneten je Fraktion zeigt, dass parteiübergreifend weder der Mandatstyp noch parlamentarische Sozialisation einen linearen Effekt auf das Abstimmungsverhalten haben. Allerdings weichen Abgeordnete mit einer hohen Expertise in der Europapolitik häufiger von der Parteilinie ab als ihre fachfremden Kollegen. Dieses Ergebnis verstärkt den Eindruck, dass die finanz- und wirtschaftspolitischen Fachkenntnisse vieler Parlamentarier den ausgedehnten Mitwirkungsrechten des Bundestages in der Euro-Krise (noch) hinterherhinken.
\end{abstract}

\section{Einleitung 1}

Seit dem Ausbruch der Finanz- und Staatsschuldenkrise in der Eurozone (kurz: Euro-Krise) sind die Mitglieder des Deutschen Bundestages in immer kürzeren Abständen aufgerufen, über Gesetzentwürfe und Anträge der Bundesregierung zu entscheiden, die zuvor als Maßnahmen zur Euro-Rettung auf europäischer Ebene vereinbart wurden. Beginnend mit dem ersten Hilfspaket für Griechenland hat der Bundestag bis Ende 2012 über elf parlamentarische Vorlagen der christlich-liberalen Koalition namentlich abgestimmt, die in einem direkten Zusammenhang mit der Euro-Krise stehen. Diese Form der Beteiligung an Europapolitik hat viele Parlamentarier vor neue Herausforderungen gestellt, da sie sich über hochkomplexe Sachverhalte wie etwa die Europäische Finanzstabilisierungsfazilität (EFSF) eine eigene Meinung bilden und darauf vorbereitet sein mussten, ihr Abstimmungsverhalten öffentlich zu begründen.

Die große Mehrheit der Abgeordneten aller Parteien orientierte sich in dieser Situation wie üblich an den Beschlussempfehlungen ihrer jeweiligen Fraktionsspitzen. In allen Parteien außer der Linkspartei, die alle Beschlüsse des Bundestages zur Euro-Krise geschlossen abgelehnt hat, bildeten sich jedoch kleine Gruppen von Parlamentariern, die von der fraktionellen Mehrheitsmeinung abgewichen sind. Insbesondere die Abweichler in den beiden Regierungsfraktionen CDU/CSU und FDP brachten die Bundesregierung in Bedrängnis, weil die „Kanzlermehrheit“ von 311 Stimmen mehrmals verfehlt wurde. Wenn alle Abgeordneten der Oppositionsfraktionen gegen die Gesetzentwürfe der Regierung gestimmt hätten, wären die von den Staats- und Regierungschefs bereits auf den Weg gebrachten Maßnahmen zur Stabilisierung der Eurozone nicht in Kraft getreten, weil sie in Deutschland parlamentarisch ratifiziert werden müssen. Diese „Euro-Skeptiker“ haben das schärfste Schwert gezogen, das einzelnen Abgeordneten zur Verfügung steht, um ihren Unmut über die Politik der Regierung offen Ausdruck zu verleihen (Patzelt, 1999). Namentliche Abstimmungen in der Euro-Krise können somit als erklärungsbedürftiger „Härtefall“ für abweichendes Verhalten bezeichnet werden, weil sich ungewöhnlich viele Abgeordnete gerade der Regierungsparteien trotz einer äußerst knappen Mehrheitskonstellation im Bundestag nicht an die eindeutig formulierten Empfehlungen ihrer Fraktionsspitzen gehalten haben.

Dieser Artikel fokussiert sich auf namentliche Abstimmungen zur Euro-Krise im Deutschen Bundestag und testet im direkten Vergleich mit den zustimmenden Abgeordneten der jeweiligen Fraktionen drei Erklärungsmodelle für abweichendes Verhalten. Der folgende zweite Abschnitt umreist kurz die Mitwirkungs- und Beteiligungsrechte des Bundestages in der Euro-Krise und gibt einen Überblick über die bisherigen Gesetze und Beschlüsse, über die namentlich abgestimmt wurde. Im dritten Abschnitt werden dann die Ergebnisse dieser Abstimmungen bis Ende 2012 dokumentiert und eine Gruppe von Abweichlern bestimmt, die gegen die Mehrheitsmeinung ihrer Fraktionen votiert haben. Darauf aufbauend werden im vierten Abschnitt drei Einflussfaktoren theoretisch hergeleitet und empirisch getestet, die dieses abweichende Stimmverhalten möglicherweise erklären können: der Mandatstyp (Direktmandat/Listenmandat), parlamentarische Sozialisation (Anzahl an Legislaturperioden) und die fachliche Expertise der Abgeordneten. Abschließend werden die zentralen Ergebnisse zusammengefasst und einige Schlussfolgerungen zur Rolle von Politikberatung in Zeiten der Euro-Krise diskutiert.

\section{Die Rolle des Deutschen Bundestages in der Euro-Krise}

Hinter dem Schlagwort „Euro-Krise“ verbirgt sich gleich eine ganze Reihe von wirtschafts-und finanzpolitischen Problemen in der Eurozone, die eng mit der hohen Staatsverschuldung einiger EU-Mitgliedstaaten verbunden sind und nicht isoliert

\footnotetext{
1 Dieser Aufsatz enthält Ergebnisse des Projekts „Innerparteilicher Konflikt in der Euro-Krise“, das an der Arbeitsgruppe „Politisches System Deutschlands und der EU “ am Institut für Sozialwissenschaften der Universität Oldenburg unter meiner Leitung (10/2012-09/2013) durchgeführt wurde. Der Autor dankt den studentischen Mitarbeitern Carina Oedingen und Sascha Gropp für ihre Unterstützung bei der Datenerhebung und Datenauswertung.
} 
voneinander betrachtet werden können (Seidel, 2010). Einschließlich der ersten Gewährung von Hilfskrediten an Griechenland, die mit dem Währungsunion-Finanzstabilitätsgesetz am 7. Mai 2010 beschlossen wurden (Kunstein \& Wessels, 2011), hat der Bundestag bis Ende 2012 über nicht weniger als elf Gesetzentwürfe und Anträge der Bundesregierung namentlich abgestimmt, mit denen diverse Euro-Rettungsmaßnahmen, die zuvor auf europäischer Ebene vereinbart wurden, umgesetzt werden sollten (s. Tabelle 1 im Anhang). Diese hohe parlamentarische Aktivität ist nicht zuerst auf die Bedeutung der Euro-Krise selbst zurückzuführen, sondern begründet sich mit dem Rechtscharakter der meisten Maßnahmen, die nicht auf Basis der geltenden EU-Verträge vereinbart wurden, sondern überwiegend auf völkerrechtlichen Verträgen und zwischenstaatlichen Abkommen beruhen, die grundsätzlich der Zustimmung des Bundestages bedürfen (Müller-Graff, 2011). Für eine Änderung der vertraglichen Grundlagen der EU oder bei Gründung neuer supranationaler Einrichtungen wie z. B. dem Europäischen Stabilitätsmechanismus (ESM) ist gemäß Art. 23 Abs. 1 GG sogar eine Zweidrittelmehrheit in Bundestag und Bundesrat notwendig (Hufeld, 2011, S. 127).

Die beiden bislang folgenreichsten Entscheidungen in der Euro-Krise waren die Einrichtung des dauerhaften Europäischen Stabilitätsmechanismus (ESM) und der Vertrag über Stabilität, Koordinierung und Steuerung in der Wirtschaftsund Währungsunion, kurz „Fiskalpakt“. Der ESM ist eine internationale Finanzinstitution mit Sitz in Luxemburg, die im Juni 2013 die Europäische Finanzstabilisierungsfazilität (EFSF) abgelöst hat und langfristig zur Stabilisierung der Eurozone beitragen soll. Der ESM kann Bürgschaften übernehmen und zinsgünstige Kredite an überschuldete Mitgliedsstaaten der Eurozone vergeben, um deren Zahlungsunfähigkeit zu verhindern. Er finanziert sich aus Kreditzusagen der EU-Mitgliedstaaten und verfügt über ein Haftungsvolumen von insgesamt 700 Milliarden Euro. Der deutsche Anteil daran beläuft sich auf rund 190 Milliarden Euro. Das oberste Entscheidungsgremium des ESM ist der Gouverneursrat, der sich aus den für Finanzen zuständigen Ministern der Mitgliedsländer zusammensetzt. ${ }^{2}$ Allerdings hat das Bundesverfassungsgericht in seinem Urteil zum ESM vom 12. September 2012 festgelegt, dass der Bundestag konsultiert werden muss, wenn die Finanzminister über neue Hilfen für ein Euroland, über Änderungen des Stammkapitals oder über das maximale Kreditvolumen, das Deutschland bereitstellt, entscheiden. ${ }^{3}$

Der Fiskalpakt ist ein zwischenstaatlicher Vertrag, mit dem sich alle EU-Mitgliedstaaten (außer Großbritannien und Tschechien) gegenseitig zu einer stärkeren Haushaltsdisziplin verpflichten. Ein zentraler Bestandteil ist die Verankerung einer Schuldenbremse in den nationalen Rechtsordnungen. Diese soll dazu beitragen, das strukturelle Staatsdefizit mittelfristig auf 0,5 Prozent des Bruttoinlandsprodukts (BIP) zu begrenzen, solange die Schuldenquote nicht deutlich unter 60 Prozent der Wirtschaftsleistung liegt. Zudem wurde das Defizitverfahren des Stabilitäts- und Wachstumspaktes von 1997 verschärft: Wenn das Staatsdefizit mehr als drei Prozent des BIP beträgt, sieht der Fiskalpakt nun vor, dass die Mitgliedstaaten die Einleitung eines Verfahrens sowie die mögliche Verhängung von Strafzahlungen als Ultima Ratio nur noch dann abwenden können, wenn sich der Rat mit qualifizierter Mehrheit dagegen ausspricht (Calliess \& Schoenfleisch, 2012, S. 482). ${ }^{4}$

Im Schatten der Euro-Krise wurden zudem neue Formen der differenzierten Integration geschaffen („Eurogipfel“) und bestehende Gremien wie z.B. die Eurogruppe mit weitreichenderen Entscheidungsbefugnissen ausgestattet (Ondarza, 2013). Politische Maßnahmen, die innerhalb solcher Koordinationsformen ausverhandelt werden, haben jedoch im Gegensatz zu EU-Rechtsakten in der Regel keine Direktwirkung, d.h. sie begründen nicht schon durch einen Beschluss auf europäischer Ebene eine geltende Rechtsnorm (Hölscheidt, 2013). Somit hat der Bundestag in der Euro-Krise mehr Kontroll- und Mitbestimmungsrechte als im ordentlichen EU-Gesetzgebungsverfahren, weil u.a. jede Änderung und Erweiterung der Euro-Rettungsschirme von den Abgeordneten bestätigt werden muss, bevor diese in Kraft treten können (Klein, 2012, S. 223). Der Bundestag ist damit in der Euro-Krise zumindest unter rein prozessualen Gesichtspunkten zu einem ernstzunehmenden Vetospieler aufgestiegen - ein Umstand, der grundsätzlich auch die Rolle der parlamentarischen Opposition in europapolitischen Fragen stärken könnte (vgl. dazu Holzhacker, 2005).

\section{Namentliche Abstimmungen in der Euro-Krise (2010-2012)}

Im Normalfall wird im Plenum des Bundestages durch Handzeichen abgestimmt, nur bei Schlussabstimmungen über Gesetzentwürfe erfolgt die Abstimmung durch Aufstehen bzw. Sitzenbleiben. Namentliche Abstimmungen müssen gemäß $\$ 52$ der Geschäftsordnung des Deutschen Bundestages bis zur Eröffnung der Abstimmung von einer Fraktion oder von anwesenden fünf Prozent der Mitglieder des Bundestages verlangt werden, d.h. wenn niemand eine namentliche Abstimmung beim Bundestagspräsidenten beantragt, wird das Abstimmungsverhalten der einzelnen Abgeordneten nicht erfasst. Einfache Abstimmungen per Akklamation wären demnach bei allen Gesetzen und Anträgen der Bundesregierung zur Euro-Krise trotz der Reichweite der Beschlüsse rechtlich zulässig gewesen. Welche Fraktionen namentliche Abstimmungen beantragt haben, ist den Plenarprotokollen in der Regel nicht $\mathrm{zu}$ entnehmen und wird vom Bundestag auch nicht offiziell dokumentiert. ${ }^{5}$

2 Vgl. Vertrag zur Einrichtung des Europäischen Stabilitätsmechanismus (ESM), der am 23. Januar 2012 von den Finanzministern der Euro-Staaten beschlossen wurde und am 27. September 2012 in Kraft getreten ist. 3 BVerfG, 2 BvR 1390/12 vom 12.9.2012, Absatz-Nr. (1 - 319), http://www. bverfg.de/entscheidungen/rs20120912_2bvr139012.html.

4 Vgl. Vertrag über Stabilität, Koordinierūng und Steuerung in der Wirtschafts- und Währungsunion, der am 2. März 2012 von 25 Mitgliedstaaten der EU unterzeichnet wurde und am 1. Januar 2013 in Kraft getreten ist.

5 Anfrage beim telefonischen Auskunftsdienst des Bundestages vom 2. Juli 2013. 


\begin{tabular}{|c|c|c|c|c|c|c|c|c|c|c|}
\hline & Gesetz/Antrag & Verfahren & Datum & $\mathrm{CDU} / \mathrm{CSU}$ & SPD & FDP & $\begin{array}{l}\text { Bgo/ } \\
\text { Grüne }\end{array}$ & $\begin{array}{l}\text { Die } \\
\text { Linke }\end{array}$ & $\begin{array}{c}\text { Gesamt } \\
\text { (JA/NEIN/Enthaltung) }\end{array}$ & $\begin{array}{c}\text { "Kanzlermehrheit" } \\
\text { (311 Stimmen) }\end{array}$ \\
\hline 1 & WFStC & EM & 7.5 .2010 & $234 / 4 / 0$ & 4/0/134 & $92 / 1 / 0$ & $61 / 0 / 5$ & o/67/o & $391 / 72 / 139$ & $\mathrm{Ja}(326)$ \\
\hline 2 & StabMechG & $E M$ & 21.5 .2010 & $230 / 4 / 3$ & $\mathrm{o} / 1 / 128$ & $89 / 2 / 1$ & $0 / 0 / 63$ & o/66/o & $319 / 73 / 195$ & Ja (319) \\
\hline 3 & 1. Änderung StabMechG & $E M$ & 29.9.2011 & $226 / 10 / 1$ & $141 / 1 / 1$ & $89 / 3 / 1$ & $67 / 1 / 0$ & o/70/0 & $523 / 85 / 3$ & $\mathrm{Ja}(315)$ \\
\hline 4 & Entschließungsantrag & $E M$ & 26.10 .2011 & $224 / 9 / 1$ & $129 / 4 / 3$ & $87 / 5 / 0$ & $63 / 1 / 0$ & o/70/o & $503 / 89 / 4$ & $\mathrm{Ja}(311)$ \\
\hline 5 & Finanzhilfe Griechenland & $E M$ & 27.2 .2012 & $219 / 13 / 2$ & $129 / 7 / 1$ & $85 / 4 / 1$ & $63 / 0 / 1$ & o/66/o & $496 / 90 / 5$ & Nein (304) \\
\hline 6 & "Fiskalpakt" & $\mathrm{QM}$ & 29.6 .2012 & $231 / 3 / 1$ & $116 / 23 / 1$ & $87 / 4 / 1$ & $54 / 9 / 3$ & $\mathrm{o} / 71 / \mathrm{o}$ & $488 / 110 / 6$ & $\mathrm{Ja}(318)$ \\
\hline 7 & „ESM-Gesetz" & QM & 29.6 .2012 & $218 / 16 / 1$ & $128 / 8 / 4$ & $82 / 10 / 0$ & $65 / 1 / 0$ & o/71/o & $493 / 106 / 5$ & Nein (300) \\
\hline 8 & ESMFinG & $E M$ & 29.6 .2012 & 218/16/1 & $129 / 5 / 4$ & $83 / 8 / 0$ & $65 / 1 / 0$ & $0 / 71 / 0$ & $495 / 101 / 5$ & Nein (301) \\
\hline 9 & „Änderung Art. 136“ & $E M$ & 29.6 .2012 & 218/16/1 & $138 / 1 / 0$ & $83 / 8 / 0$ & $65 / 1 / 0$ & $\mathrm{O} / 71 / 0$ & $504 / 97 / 1$ & Nein (301) \\
\hline 10 & Finanzhilfe Spanien & $E M$ & 19.7 .2012 & $221 / 13 / 0$ & $118 / 14 / 2$ & $80 / 9 / 1$ & $54 / 1 / 10$ & o/6o/o & $473 / 97 / 13$ & Nein (301) \\
\hline 11 & 2. Änderung StabMechG & $E M$ & 30.11 .2012 & $217 / 12 / 1$ & $111 / 11 / 9$ & $80 / 10 / 0$ & $65 / 0 / 1$ & o/67/o & $473 / 100 / 11$ & Nein (297) \\
\hline
\end{tabular}

Quelle

Eigene Auszählung auf Basis DIP (Dokumentations- und Informationssystem für Parlamentarische Vorgänge) des Deutschen Bundestages (http://dipbt.bu ndestag.de); endgültige Plenarprotokolle 17/41 (7.5.2010), S. 4019-4022; $17 / 44$ (21.5.2010), S. 4443-4445; 17/130 (29.9.2011), S. 15234-15239; 17/135 (26.10.2011), S. 15978-15980; $17 / 160$ (27.2.2012), S. 19105-19107; 17/188 (29.6.2012), S. 22736-22739, 22740-22742, 22745-22747, 17/189 (19.7.2012), S. 22836-22839; 17/206 (20.11.2012), S. 25135; 17/212 (30.11.2012), S. 25991-25993

Anmerkungen

Verfahren: EM = einfache Mehrheit der abgegebenen Ja-Stimmen; QM = Qualifizierte Mehrheit (absolute Zweidrittelmehrheit gemäß Art. 79 Abs. 2 GC = 414 Stimmen); „Kanzlermehrheit“ = Abgegebene Ja-Stimmen von mind. der Hälfte aller Mitglieder des Deutschen Bundestages plus eine Stimme durch Abgeordnete der Regierungskoalition, derzeit 311 Stimmen)

Tabelle 2 zeigt die Ergebnisse der namentlichen Abstimmungen der fünf Bundestagsfraktionen in der Euro-Krise. Zunächst ist festzuhalten, dass alle elf Gesetzentwürfe bzw. Anträge der christlich-liberalen Bundesregierung mit deutlichen Mehrheiten angenommen wurden. Lediglich auf die ersten beiden Gesetzentwürfe entfielen weniger Ja-Stimmen, weil sich fast die gesamte Fraktion der SPD enthalten hat; die Fraktion Bündnis 90/Die Grünen enthielt sich demgegenüber nur in der zweiten Abstimmung über das Stabilisierungsmechanismusgesetz vom 21. Mai 2010, mit dem vor allem die deutschen Haftungsgarantien für die EFSF auf 211 Milliarden Euro angehoben wurden (Belke \& Dreger, 2011). Allein Die Linke hat an ihrer konsequenten Opposition gegen die ihrer Auffassung nach zu wirtschaftsliberale Ausrichtung der deutschen Europapolitik festgehalten und bislang alle Euro-Rettungsmaßnahmen der Bundesregierung geschlossen abgelehnt. Das Konsensprinzip in der deutschen Europapolitik besteht also zumindest unter CDU/CSU, FDP, SPD und Bündnis 90/Die Grünen weiter, auch wenn sich der Konflikt über inhaltliche Lösungsstrategien zwischen den Regierungs- und Oppositionsparteien in der Euro-Krise deutlich intensiviert hat (siehe dazu Wimmel, 2012).

Obwohl die Führungen aller Bundestagsfraktionen außer der Linkspartei ihre Abgeordneten bei den allermeisten Abstimmungen dazu ermutigt haben, die auf europäischer Ebene vereinbarten Maßnahmen mit ihrer Stimme zu unterstützen, formierten sich abgesehen von der Linkspartei in allen Fraktion kleinere Gruppen von Parlamentariern, die sich den Beschlussempfehlungen ihrer Parteispitzen widersetzt und gegen die Mehrheitsmeinung in ihren Fraktionen gestimmt haben. In der CDU/CSU-Fraktion hat sich die Zahl der
Abweichler von anfangs vier auf zeitweilig bis zu 16 Abgeordnete erhöht, die u.a. das wichtige Gesetz zur Umsetzung des ESM nicht mittragen wollten. Eine ähnliche Entwicklung hat in der FDP-Fraktion stattgefunden, wo sich eine Gruppe von Abweichlern nach und nach um den Abgeordneten Frank Schäffler versammelte und in den letzten Abstimmungen bis zu zehn Liberale mit Nein stimmten. ${ }^{6}$ In der SPD-Fraktion haben sich ebenfalls Gruppen von Abweichlern gebildet: In der ersten Abstimmung votierten vier Abgeordnete mit Ja, obwohl sich ansonsten die gesamte Fraktion enthielt. Nach dem Schwenk der SPD-Führung, sich fortan bei Abstimmungen zur Euro-Krise nicht länger enthalten, sondern die Hilfsmaßnahmen der Bundesregierung trotz weiterhin bestehender inhaltlicher Differenzen unterstützen zu wollen, formierte sich eine neue Abweichlergruppe, die diesen Kurswechsel nicht mittragen wollte. Der größte Widerstand gegen die Fraktionslinie bildete sich bei der Abstimmung über den „Fiskalpakt“ am 29. Juni 2012, als 23 SPD-Abgeordnete mit Nein stimmten. Bündnis 90/Die Grünen traten bei den meisten Abstimmungen sehr geschlossen auf, nur dem „Fiskalpakt“ verweigerten neun Abgeordnete ausdrücklich ihre Zustimmung und zehn Parlamentarier enthielten sich bei dem Beschluss über die Gewährung von Finanzhilfen für Spanien. Die größte Geschlossenheit zeigte die Fraktion Die Linke, die bei allen Entscheidungen zur Euro-Krise einstimmig gegen die

6 In einer von Schäffler initiierten Mitgliederbefragung wurde die Einrichtung des dauerhaften Euro-Schutzschirms ESM und damit der europapolitische Kurs des FDP-Bundesvorstands von 54,5 Prozent der gültigen abgegebenen Stimmen unterstützt. Vgl. Frankfurter Allgemeine Zeitung: Knapper Sieg des FDP-Vorstands über Euro-Skeptiker, 17.12.2011. 


\begin{tabular}{|c|c|c|c|c|c|c|c|}
\hline \multirow[t]{2}{*}{ Fraktion } & \multirow[t]{2}{*}{ Abgeordnete gesamt } & \multicolumn{2}{|c|}{$\begin{array}{l}\text { Abweichler je } \\
\text { Fraktion }\end{array}$} & \multicolumn{4}{|c|}{ Anzahl Abweichungen } \\
\hline & & $\mathrm{N}$ & $\%$ & $1-2$ & $3-5$ & $6-8$ & 9-11 \\
\hline CDU/CSU & 237 & 23 & 9,7 & 6 & 6 & 6 & 5 \\
\hline SPD & 146 & 35 & 24,0 & 19 & 11 & 4 & 1 \\
\hline FDP & 93 & 14 & 15,1 & 5 & 2 & 5 & 2 \\
\hline Die Linke & 76 & o & - & o & o & o & o \\
\hline B9o/Grüne & 68 & 17 & 25,0 & 14 & 2 & o & 1 \\
\hline Gesamt & 620 & 89 & 14,4 & 44 & 21 & 15 & 9 \\
\hline
\end{tabular}

Tabelle 3: Abweichler und Anzahl der Abweichungen nach Fraktion

Quelle: Eigene Auszählung auf Basis DIP (Dokumentations- und Informationssystem für Parlamentarische Vorgänge) des Deutschen Bundestages (http:// dipbt.bundestag.de); endgültige Plenarprotokolle s. Tabelle 2; Abgeordnete gesamt: Abgeordnete nach Fraktionen (http://www.bundestag.de/bundestag/ abgeordnete17/listeFraktionen/index.html); Stand: 03/2013

Beschlussvorlagen der Bundesregierung votiert hat, ohne dass auch nur ein einziger Abgeordneter einmal von dieser Linie abgewichen wäre.

Tabelle 3 listet die Anzahl an Abweichlern sowie die Anzahl der Abweichungen nach Fraktion auf. Abweichler sind definiert als Abgeordnete, die mindestens bei einer der elf Abstimmungen zur Euro-Krise gegen die Fraktionsmehrheit votiert haben. Einbezogen wurden folglich auch Enthaltungen, wenn die anderen Abgeordneten der Fraktion mehrheitlich mit Ja oder Nein gestimmt haben. Daraus ergibt sich eine Gruppe von insgesamt 89 Abweichlern. Die anteilig meisten Abweichler befinden sich in der Fraktion von Bündnis 90/Die Grünen: 17 der 68 Grünen-Abgeordneten wichen mindestens einmal von der Fraktionslinie ab, was einem Anteil von 25 Prozent entspricht. Knapp dahinter folgt die SPD-Fraktion mit 35 Abweichlern (24 Prozent) vor der FDP-Fraktion mit 14 Abweichlern (15,1 Prozent). Die geringste Abweichlerquote findet sich mit 9,7 Prozent in der CDU/CSU-Fraktion, was aufgrund der Fraktionsstärke von 237 lediglich 23 Abgeordnete ausmacht. Neun dieser 23 Abweichler gehören der Schwesterpartei CSU an, so dass gemessen an den nur 44 CSUAbgeordneten im Bundestag hier ein starkes Übergewicht vorliegt. In den beiden Regierungsfraktionen waren also proportional gesehen weniger Abweichler als in den rot-grünen Oppositionsfraktionen. Dieses Ergebnis entspricht auf den ersten Blick den Erwartungen, weil die Regierung mehr auf die Unterstützung „ihrer“ Parlamentsfraktionen angewiesen ist als die Opposition, um handlungsfähig zu sein und Gesetzesvorhaben realisieren zu können.

Die rechte Spalte zeigt die Anzahl an Abweichungen, also wie oft die Abgeordneten jeweils von ihrer Mehrheitsfraktion abgewichen sind. Diese Daten legen einige Unterschiede zwischen den Regierungs- und Oppositionsfraktionen frei, die den ersten Eindruck relativieren: In den Fraktionen von CDU/ CSU und FDP sind die einzelnen Abgeordneten wesentlich öfter abgewichen als bei SPD und Bündnis 90/Die Grünen. Sechs der 23 CDU/CSU-Abweichler sind 6-8 Mal abgewichen und fünf von ihnen kommen sogar auf 9-11 Abweichungen (3 sind bei allen 11 Abstimmungen abgewichen). Ein ähnliches Verhältnis zeigt sich in der FDP-Fraktion, wo fünf der 14
Abweichler 6-8 Mal abgewichen sind und zwei von ihnen 9-11 Mal gegen die Beschlussvorlagen der Bundesregierung stimmten (der Wortführer der „Euro-Skeptiker“ in der FDP-Fraktion, Frank Schäffler, stimmte als einziger immer mit Nein). In den rot-grünen Oppositionsfraktionen stellt sich das Bild anders dar, hier finden sich deutlich mehr Abgeordnete, die nur bei einigen ausgewählten Abstimmungen von der Fraktionslinie abgewichen sind: Neunzehn der 35 SPD-Abweichler sind nur bei 1-2 Abstimmungen abgewichen und lediglich ein Abgeordneter der SPD-Fraktion reiht sich in die Gruppe der permanenten Abweichler (9-11) ein. Noch ungleicher ist die Verteilung in der Fraktion von Bündnis 90/Die Grünen, denn 14 der 17 Abweichler sind nur 1-2 Mal abgewichen. Der Hintergrund dafür ist, dass viele Abweichler aus den Oppositionsfraktionen nur den umstrittenen „Fiskalpakt“ abgelehnt haben, ansonsten aber den diversen anderen Regierungsvorlagen zur Euro-Krise zustimmten und damit der Parteilinie gefolgt sind.

In den Regierungsfraktionen war die Gruppe der „Kettenabweichler“ also deutlich größer als in den Oppositionsfraktionen, in denen viel mehr „Gelegenheitsabweichler“ zu finden sind. Diese Aufteilung führt dazu, dass die 23 CDU/CSUAbweichler auf insgesamt 117 abweichende Stimmen in allen Abstimmungen kommen (FDP: 14 Abweichler/69 abweichende Stimmen), während die 35 SPD-Abweichler zusammen nur 104 abweichende Stimmen auf sich vereinen und auf die 17 Grünen-Abweichler sogar insgesamt nur 35 abweichende Stimmen entfallen. Insgesamt sind 5,2 Prozent aller abgegebenen Stimmen der CDU/CSU-Abgeordneten von der Fraktionslinie abgewichen, in der SPD-Fraktion waren es 7,4 Prozent, bei der FDP ebenfalls 7,4 Prozent und bei Bündnis 90/ Die Grünen nur 5,1 Prozent. Gemessen am proportionalen Anteil an abweichenden Stimmen haben die Regierungsfraktionen folglich nicht geschlossener abgestimmt als die Oppositionsfraktionen.

\section{Abweichendes Abstimmungsverhalten in der Euro-Krise}

Der Deutsche Bundestag zeichnet sich wie die meisten legislativen Körperschaften in parlamentarischen Regierungssystemen grundsätzlich durch eine hohe Fraktionsdisziplin aus (vgl. 
Saalfeld, 1995; Patzelt, 2003; Sieberer, 2006). Das in Art. 38 GG festgeschriebene freie Mandat, wonach Mitglieder des Bundestages „an Aufträge und Weisungen nicht gebunden und nur ihrem Gewissen unterworfen “ sind, steht also in der Praxis einer großen innerfraktionellen Geschlossenheit im Abstimmungsverhalten nicht entgegen, sondern gerade Abgeordnete der Regierungsparteien unterstützen in der Regel die Gesetzentwürfe „ihrer“ Regierung (Dittberner, 2003). Dafür gibt sowohl politische als auch strategische Motive: Einerseits sollte eine gewisse ideologische Nähe zu der Partei angenommen werden, der Abgeordnete angehören und für die sie ein Mandat errungen haben; andererseits sind Parlamentarier natürlich von dem Wohlergehen und Wohlwollen ihrer Partei abhängig, sodass sie kein Interesse an einer eventuellen vorzeitigen Auflösung des Bundestages, etwa nach einer gescheiterten Vertrauensfrage, haben bzw. bei der kommenden Wahl nur dann auf einen aussichtsreichen Listenplatz hoffen können, wenn sie sich innerparteilich kooperativ verhalten (Saalfeld, 2005).

Dementsprechend ist es nicht überraschend, dass auch in der Euro-Krise die ganz überwiegende Mehrheit der Mitglieder des Bundestages entlang den Beschlussempfehlungen ihrer Parteispitzen abgestimmt hat. Etwas mehr als 85 Prozent der gesamten Abgeordneten hielten sich bei allen elf namentlichen Abstimmungen an die vorher ausgegebene Parteilinie. Trotzdem fällt die relativ hohe und konstante Zahl an Abweichlern insbesondere im Regierungslager auf, die trotz klar formulierter Kooperationserwartung durch die Partei- und Fraktionsvorsitzenden keine Bereitschaft gezeigt haben, die von der Bundesregierung maßgeblich vorangetriebenen Beschlüsse zur Euro-Rettung mitzutragen. Erschwerend kommt noch hinzu, dass die christlich-liberale Bundesregierung keineswegs über ein solides Stimmenpolster im Bundestag verfügte, sodass einige Stimmen mehr oder weniger kaum ins Gewicht gefallen wären. Durch die Abweichler im Regierungslager konnte die sogenannte „Kanzlermehrheit“ von 311 Stimmen in sechs von elf Abstimmungen nicht erreicht werden, d.h. die Regierung war auf Stimmen aus den Oppositionsfraktionen angewiesen, um die erforderlichen Mehrheiten für ihre Euro-Rettungspolitik zu finden.

Während sich die meisten Studien auf die Erklärung innerfraktioneller Geschlossenheit konzentriert haben, liegen nur wenige vergleichende Analysen zu den Determinanten abweichenden Stimmverhaltens in parlamentarischen Regierungssystemen vor (vgl. Fritzsche, 2009). Dieser lückenhafte Forschungsstand wirft die Frage auf, ob und inwieweit sich gemeinsame Merkmale finden lassen, durch die sich die Gruppe der Abweichler von den zustimmenden Abgeordneten in den jeweiligen Fraktionen unterscheidet? Im Folgenden werden zunächst zwei strukturelle Einflussfaktoren für abweichendes Abstimmungsverhalten am Fall der Euro-Krise kontrolliert, die immer wieder als mögliche Erklärungsmodelle herangezogen werden, nämlich der Mandatstyp (Direktmandat/Listenmandat) und parlamentarische Sozialisation (Anzahl an Legislaturperioden). Danach wird ein dritter möglicher Einflussfaktor für abweichendes Verhalten in der Euro-
Krise theoretisch hergeleitet und getestet, der in der einschlägigen Literatur bislang noch keine Beachtung gefunden hat: die fachliche Expertise der Abgeordneten in der Europapolitik.

\section{Mandatstyp (Direktmandat/Listenmandat)}

Ein möglicher Einflussfaktor für Fraktionsgeschlossenheit bzw. für abweichendes Verhalten bei Abstimmungen ist der Mandatstyp des einzelnen Abgeordneten (vgl. u.a. Stecker 2011). Bei Bundestagswahlen wird aus jedem der 299 Wahlkreise der Kandidat mit den meisten Erststimmen direkt in den Bundestag entsendet und weitere 299 Mandate (plus Überhangmandate) werden über die Landeslisten der Parteien je nach Zweitstimmenanteil vergeben. Abgeordnete mit einem Direktmandat sind mit Blick auf einen möglichen Wiedereinzug in den Bundestag strukturell unabhängiger von ihrer Partei als Abgeordnete, die ihr Mandat über Landeslisten errungen habe. Erstens erfolgt die Wahl zur Aufstellung von Direktkandidaten ausschließlich im jeweiligen Wahlkreis und damit in einem kleineren Kreis von Parteifreunden, während sich Listenkandidaten auf großen Landesparteitagen zur Wahl stellen müssen, um einen der wenigen aussichtsreichen Listenplätze zu erreichen (Schüttemeyer, 2002). ${ }^{7}$ Diese Konstellation lässt zumindest vermuten, dass Abgeordnete mit Direktmandat, die mit ihrem Wahlkreis in der Regel eng verwurzelt sind, weniger für abweichendes Stimmverhalten sanktioniert werden als Listenkandidaten. Zweitens hängt eine Wiederwahl von Direktkandidaten viel stärker von der Unterstützung der Wähler im Wahlkreis ab, sodass sich abweichendes Verhalten sogar auszahlen kann, wenn die Parteiposition bei den Bürgern unpopulär ist. Zudem können sich Wahlkreiskandidaten gemäß $\$ 34$ der Bundeswahlordnung (BWO) auch unabhängig von ihrer Partei um ein erneutes Direktmandat bewerben, wenn sie 200 Unterstützungsunterschriften von Wahlberechtigten des Wahlkreises vorweisen, während Listenkandidaten ausschließlich nach geheimer Wahl von der Partei nominiert werden. Diese Faktoren zusammengenommen könnten den Druck zu parteikonformen Verhalten bei Direktmandataren geringer erscheinen lassen als bei Abgeordneten, die vor der nächsten Wahl auf eine aussichtsreiche Listenplatzierung angewiesen sind. So hat eine statistische Analyse zum Abstimmungsverhalten im Bundestag während der Großen Koalition (2005-2009) ergeben, dass Abgeordnete mit Direktmandat bei namentlichen Abstimmungen signifikant häufiger von der Parteilinie abgewichen sind als Listenabgeordnete (Sieberer, 2010). Dementsprechend geht das erste Erklärungsmodell davon aus, dass Abgeordnete, die mit einem Direktmandat in den Bundestag eingezogen sind, auch in der Euro-Krise eher

7 Gemäß § 21(1) BWG kann als Bewerber einer Partei in einem Kreiswahlvorschlag nur benannt werden, wer nicht Mitglied einer anderen Partei ist und in einer Mitgliederversammlung zur Wahl eines Wahlkreisbewerbers gewählt worden ist. Eine „Mitgliederversammlung zur Wahl eines Wahlkreisbewerbers ist eine Versammlung der im Zeitpunkt ihres Zusammentritts im Wahlkreis zum Deutschen Bundestag wahlberechtigten Mitglieder der Partei“. 


\begin{tabular}{|c|c|c|c|c|c|c|c|}
\hline \multirow[t]{2}{*}{ Fraktion } & \multirow[t]{2}{*}{$\begin{array}{l}\text { Abgeordnete (Direktmandat/ } \\
\text { Listenmandat) }\end{array}$} & \multicolumn{2}{|c|}{$\begin{array}{l}\text { Abweichler je } \\
\text { Fraktion }\end{array}$} & \multicolumn{2}{|c|}{$\begin{array}{c}\text { Abweichler } \\
\text { Direktmandat }\end{array}$} & \multicolumn{2}{|c|}{$\begin{array}{c}\text { Abweichler } \\
\text { Listenmandat }\end{array}$} \\
\hline & & $\mathrm{N}$ & $\%$ & $\mathrm{~N}$ & $\%$ & $\mathrm{~N}$ & $\%$ \\
\hline CDU/CSU & $237(216 / 21)$ & 23 & 9,6 & 22 & 10,2 & 1 & 4,8 \\
\hline SPD & $146(64 / 82)$ & 35 & 24,0 & 9 & 6,2 & 26 & 31,7 \\
\hline FDP & 93 (0/93) & 14 & 15,1 & o & - & 14 & 15,1 \\
\hline Die Linke & $76(16 / 60)$ & 0 & - & o & - & 0 & - \\
\hline B9o/Grüne & $68(1 / 67)$ & 17 & 25,0 & 1 & 100 & 16 & 23,9 \\
\hline Gesamt & $620(297 / 323)$ & 89 & 14,4 & 32 & 10,8 & 57 & 17,7 \\
\hline
\end{tabular}

Tabelle 4: Einfluss Mandatstyp auf abweichendes Stimmverhalten

Quelle: Eigene Auszählung auf Basis DIP (Dokumentations- und Informationssystem für Parlamentarische Vorgänge) des Deutschen Bundestages (http:// dipbt.bundestag.de); endgültige Plenarprotokolle s. Tabelle 2; Direktmandat/Listenmandat: Abgeordnete nach Fraktionen (http://www.bundestag.de/bun destag/abgeordnete17/listeFraktionen/index.html); Stand: 03/2013

Anmerkung: Die Spaltenprozente in „Abweichler Direktmandat“ und „Abweichler Listenmandat“ beziehen sich auf die gesamten Direktmandate/Listenmandate je Fraktion; die Prozentangaben in der untersten Zeile beziehen sich auf die Gesamtzahl aller Direktmandate/Listenmandate je Fraktion inkl. der Abgeordneten der Fraktion Die Linke

zu abweichendem Verhalten neigen als Abgeordnete mit Listenmandat.

Tabelle 4 zeigt den Einfluss des Mandatstyps auf abweichendes Stimmverhalten in der Euro-Krise. Von den 23 Abweichlern in der CDU/CSU-Fraktion hatten 22 ein Direktmandat, was einem Anteil von 10,2 Prozent an allen CDU/ CSU-Abgeordneten mit Direktmandat $(\mathrm{N}=216)$ entspricht; der eine Abweichler mit Listenmandat entspricht einem Anteil von nur 4,8 Prozent, gemessen an allen CDU/CSU-Abgeordneten dieses Mandatstyps ( $\mathrm{N}=21$ ). Bei der SPD-Fraktion zeigt sich ein ganz anderes Bild: Nur 9 der 35 Abweichler hatten ein Direktmandat (6,2 Prozent), während die deutliche Mehrheit von 26 Abweichlern über Parteilisten in den Bundestag eingezogen ist (31,7 Prozent aller SPD-Listenmandate). Für die Fraktionen von FDP und Bündnis 90/Die Grünen ist ein aussagekräftiger Vergleich nicht möglich, weil die Liberalen über kein Direktmandat verfügen und die Grünen nur einen Wahlkreis direkt gewonnen haben. Insgesamt gehören 10,8 Prozent aller Abgeordneten mit Direktmandat zur Gruppe der Abweichler, während 17,7 Prozent aller Listenabgeordneten mindestens bei einer Abstimmung zur Euro-Krise von der Fraktionslinie abgewichen sind.

Der Mandatstyp hat also nur bei der CDU/CSU-Fraktion den erwarteten Effekt, weil prozentual etwas mehr Abgeordnete mit Direktmandat abgewichen sind als ihre Kollegen mit Listenmandat. Zwar muss relativierend hinzugefügt werden, dass die 216 Direktmandate in der CDU/CSU-Fraktion deutlich überwiegen, aber die wenigen Abgeordneten mit Listenmandat haben sich fast komplett parteikonform verhalten. Tatsächlich sind insbesondere CSU-Abgeordnete, die ja einen beträchtlichen Anteil an den Abweichlern ausmachen, in der Regel nicht von einer Platzierung auf der Landesliste abhängig, weil eine CSU-Wahlkreiskandidatur in Bayern erfahrungsgemäß fast schon einen Freifahrtschein in den Bundestag bedeutet und damit meist alle CSU-Bundestagsmandate vergeben sind. ${ }^{8}$ CSU-Direktkandidaten können sich also im Grunde ausschließlich darauf konzentrieren, in ihrem Wahl- kreis wiedernominiert zu werden, weil eine zusätzliche Listenplatzierung kaum wahltaktische Vorteile bringt. Bei der SPDFraktion zeigt sich jedoch ein gegenteiliger Zusammenhang: Sowohl zahlenmäßig als auch anteilig haben deutlich mehr Abgeordnete mit Listenmandat gegen die Parteilinie gestimmt, obwohl Direkt- und Listenmandate fast gleich verteilt sind. Dieser Befund ist umso bemerkenswerter, wenn man bedenkt, dass viel mehr SPD-Abgeordnete über Landeslisten in den Bundestag eingezogen sind als CDU/CSU-Abgeordnete und einkalkulieren konnten, dass sie aller Voraussicht nach auch bei der nächsten Wahl auf eine hohe Listenplatzierung angewiesen sein werden. In den Fraktionen von FDP und Bündnis 90/Die Grünen finden sich ebenfalls eine ganze Reihe von Abweichlern mit Listenmandat - wenn jedoch das Erklärungsmodell greifen würde, wäre abweichendes Stimmverhalten in diesen Fraktionen (fast) ohne Direktmandate zumindest nicht in dieser Größenordnung zu erwarten gewesen. Wahlkreisbewerbern aus diesen kleinen Parteien ist natürlich bewusst, dass sie im Normalfall nur minimale Chancen auf die Mehrheit der Erststimmen haben, sodass sie folglich nur über eine hohe Listenplatzierung in den Bundestag einziehen können. Insgesamt kann das erste Erklärungsmodell, dass sich Abgeordnete mit Direktmandat häufiger abweichendes Stimmverhalten zutrauen, weil sie hinsichtlich einer erneuten Nominierung für die nächste Legislaturperiode strukturell weniger von ihrer Partei abhängig sind als Listenkandidaten, für Abstimmungen in der Euro-Krise nicht bestätigt werden.

Eine Rollendifferenzierung nach Mandatstyp lässt sich im Abstimmungsverhalten speziell bei Gesetzen zur Euro-Krise also parteiübergreifend nicht nachweisen. Dieses Ergebnis deutet daraufhin, dass der positive Befund von Sieberer (2010) wesentlich auf die spezielle Konstellation in der Großen Koali-

8 Seit der 13. Wahlperiode (1994-1998) bis einschließlich der Bundestagswahl 2013 hat die CSU mit Ausnahme des Wahlkreises München-Nord (SPD: 1994, 1998, 2002) alle bayrischen Direktmandate errungen; vgl. Datenhandbuch des Bundestages (http://www.bundestag.de/doku mente/datenhandbuch/index.html). 


\begin{tabular}{|c|c|c|c|c|c|c|c|c|c|c|c|}
\hline \multirow[t]{3}{*}{ Fraktion } & \multirow{3}{*}{$\begin{array}{l}\text { Abweichler je } \\
\text { Fraktion }\end{array}$} & \multicolumn{10}{|c|}{ Abweichler nach Anzahl Legislaturperioden } \\
\hline & & \multicolumn{2}{|c|}{1} & \multicolumn{2}{|c|}{2} & \multicolumn{2}{|c|}{$3 / 4$} & \multicolumn{2}{|c|}{$5 / 6$} & \multicolumn{2}{|c|}{ Mehr als 6} \\
\hline & & $\mathrm{N}$ & $\%$ & $\mathrm{~N}$ & $\%$ & $\mathrm{~N}$ & $\%$ & $N$ & $\%$ & $\mathrm{~N}$ & $\%$ \\
\hline CDU/CSU & 23 & 5 & 7,6 & 4 & 14,3 & 11 & 13,9 & 3 & 6,7 & o & - \\
\hline SPD & 35 & 6 & 22,2 & 5 & 26,3 & 17 & 25,4 & 5 & 23,8 & 2 & 16,7 \\
\hline FDP & 14 & 6 & 15,0 & 4 & 19,1 & 1 & 5,2 & 2 & 18,2 & 1 & 50 \\
\hline Die Linke & 0 & 0 & - & 0 & - & 0 & - & 0 & - & 0 & - \\
\hline B9o/Grüne & 17 & 11 & 39,3 & 4 & 12,2 & 1 & 5,9 & 1 & 25 & o & - \\
\hline Gesamt & 89 & 28 & 7,1 & 17 & 15,2 & 30 & 15,7 & 11 & 12,8 & 3 & 9,7 \\
\hline
\end{tabular}

Quelle: Eigene Auszählung auf Basis DIP (Dokumentations- und Informationssystem für Parlamentarische Vorgänge) des Deutschen Bundestages (http://d ipbt.bundestag.de); endgültige Plenarprotokolle s. Tabelle 2; Anzahl Legislaturperioden: Mitgliedschaft in Wahlperioden für alle Abgeordneten (http://ww w.bundestag.de/bundestag/abgeordnete17/mdb_zahlen/mitgl_wp.html), Stand: 03/2013; Abweichler: Kürschners Volkshandbuch - 17. Wahlperiode, 126. Aufl., Neue Darmstädter Verlagsanstalt 2012

Anmerkung: Die Spaltenprozente in „Abweichler nach Anzahl Legislaturperioden“ beziehen sich auf die Gesamtzahl der Abgeordneten in der jeweiligen Legislaturperiode je Fraktion; die Prozentangaben in der untersten Zeile beziehen sich auf die Gesamtzahl aller MdBs in der jeweiligen Legislaturperiode inkl. der Abgeordneten der Fraktion Die Linke

tion zurückzuführen ist, in der die Regierung aufgrund ihrer übergroßen Parlamentsmehrheit nicht auf jede Stimme angewiesen war und gleichzeitig nahezu alle Direktmandate auf Abgeordnete der Regierungsparteien CDU/CSU und SPD entfielen. Zudem sind im bundesdeutschen Mischwahlsystem sehr viele Direktkandidaten über Landeslisten „abgesichert“, sodass eine systematische Trennung zwischen den beiden Mandatstypen generell kaum möglich ist (Manow, 2012). Darüber hinaus haben vermutlich auch nur diejenigen Direktkandidaten realistische Chancen auf einen zusätzlichen Listenplatz, die sich in der Vergangenheit parteikonform verhalten haben (Schüttemeyer \& Sturm, 2005). Folglich werden Direktkandidaten zumindest dann nicht weniger loyal gegenüber ihrer Partei auftreten als reine Listenkandidaten, wenn der Gewinn ihres Wahlkreises bei der kommenden Wahl unsicher ist und sie auf einen aussichtsreichen Listenplatz angewiesen sein könnten, um wieder in den Bundestag einzuziehen (vgl. dazu Manow \& Nistor, 2009).

\section{Parlamentarische Sozialisation (Anzahl der Legislaturperioden)}

Ein zweiter möglicher Einflussfaktor für abweichendes Abstimmungsverhalten ist parlamentarische bzw. innerfraktionelle Sozialisation (vgl. schon Sarcinelli, 1989). Junge unerfahrene Abgeordnete, so die gängige These, die noch keine etablierte Stellung unter den Parteikollegen haben, werden sich bei Abstimmungen stärker an den Beschlussempfehlungen ihrer Partei orientieren als „alte Hasen“, die seit vielen Wahlperioden ein fester Bestandteil der Fraktion sind und sich deswegen abweichendes Verhalten eher leisten können. Der Konformitätsdruck ist vermutlich wesentlich größer, wenn man als Abgeordneter neu im Bundestag sitzt und sich die Chance auf eine weitere Legislaturperiode offen halten will, als bei langgedienten Mitgliedern des Bundestages, die mit ihrer Fraktion oder ihrem Wahlkreis eng verwurzelt sind oder ggf. aus Altersgründen keine weitere Amtszeit anstreben. Fraktionen sind vermachtete Einheiten, in denen wie in allen sozialen Organisationen um Ämter und Positionen gerungen wird, auch wenn Bundestagsabgeordnete durch das Grundgesetz vor echtem Fraktionszwang umfassend geschützt sind (Isensee, 2007). Trotzdem kann angenommen werden, dass Neulinge am Anfang ihrer politischen Karriere sehr viel eher bereit sind, sich am Ende innerparteilicher Beratungsprozesse der Mehrheitsmeinung zu beugen, um sich persönliche Aufstiegschancen in der Parteihierarchie offenzuhalten, anstatt sofort auf Konfrontationskurs zu den einflussreichen Fraktionsspitzen zu gehen. Dementsprechend geht das zweite Erklärungsmodell davon aus, dass abweichendes Stimmverhalten in der Euro-Krise mit parlamentarischer Sozialisation, gemessen an der Anzahl an Legislaturperioden, ansteigt.

Tabelle 5 setzt die Anzahl an Legislaturperioden mit abweichendem Stimmverhalten in Beziehung. In der CDU/ CSU-Fraktion bestritten die meisten Abweichler ihre zweite (14,3 Prozent) und dritte/vierte Legislaturperiode (13,9 Prozent), immer prozentual gesehen zu allen CDU/CSU-Abgeordneten in der jeweiligen Wahlperiode. Die fünf CDU/CSUAbweichler in der ersten Legislaturperiode machen hingegen nur 7,6 Prozent an allen 69 Neulingen in der Fraktion aus. Allerdings sinkt die Abweichlerquote ab der fünften/sechsten Legislaturperiode wieder auf 6,7 Prozent ab und altgediente Abgeordnete mit mehr als sechs Wahlzeiten sind gar nicht von der Parteilinie abgewichen, obwohl die CDU/CSU-Fraktion in dieser Gruppe 16 Abgeordnete stellt und damit mehr als alle anderen Fraktionen. In der SPD-Fraktion zeigt sich eine fast perfekte anteilige Gleichverteilung der Abweichler zwischen den Legislaturperioden im Bereich von 22-26 Prozent, nur in der Kohorte der Abgeordneten, die bereits mehr als sechs Mal in den Bundestag gewählt worden sind, reduziert sich die Abweichlerquote leicht auf 16,7 Prozent. Auch in der FDPFraktion ist kein klarer Trend auszumachen. Von der Anzahl her befinden sich die meisten Abweichler in der ersten Wahl- 
periode, allerdings stellt die FDP in dieser Gruppe auch 40 Abgeordnete und damit anteilig mehr als alle anderen Fraktionen. Die meisten FDP-Abweichler sind mit 50 Prozent bei den Abgeordneten mit mehr als sechs Legislaturperioden, allerdings sind dieser Gruppe insgesamt nur zwei MdBs zuzuordnen. Bei Bündnis 90/Die Grünen befinden sich die mit Abstand meisten Abweichler (11) in der ersten Wahlperiode, und auch anteilig ist hier mit 39,3 Prozent fast jeder zweite Fraktionsneuling mindestens einmal von der Parteilinie abgewichen.

Die Anzahl an Legislaturperioden hat also bei keiner Fraktion den vermuteten Effekt, am ehesten noch in der CDU/CSUFraktion, in der zumindest die Novizen etwas weniger häufig abgewichen sind als die Abgeordneten in der zweiten und dritten/vierten Wahlperiode. Aber gerade die „Urgesteine“ mit mehr als sechs Wahlperioden haben sich vergleichsweise parteikonform verhalten. In der SPD-Fraktion hat parlamentarische Sozialisation überhaupt keinen Einfluss auf das Abstimmungsverhalten und in den Fraktionen der kleinen Parteien zeigt sich sogar ein gegenteiliger Zusammenhang. Die numerisch meisten Abweichler sind zum ersten Mal in den Bundestag gewählt worden und haben trotzdem häufiger gegen die Parteilinie votiert als die langgedienten Kollegen. Nimmt man alle Fraktionen zusammen, steigt abweichendes Stimmverhalten ebenfalls nicht mit der Anzahl an Legislaturperioden an, sondern fällt nach der dritten/vierten Wahlperiode wieder moderat ab auf 12,8 und 9,7 Prozent. Somit scheint langjährige parlamentarische Sozialisation parteikonformes Abstimmungsverhalten tendenziell eher zu fördern als zu schwächen. Folglich kann auch das zweite Erklärungsmodell, nach dem Abgeordnete mit weniger Jahren im Bundestag ihr Abstimmungsverhalten deutlich stärker an den Beschlussempfehlungen ihrer Fraktionsspitzen orientieren als erfahrenere Parteikollegen, nicht bestätigt werden.

Im Gegenteil: Viele junge Abgeordnete in den ersten Wahlperioden sind von der Fraktionslinie abgewichen, insbesondere bei Bündnis 90/Die Grünen und der FDP, die immerhin Regierungspartei war. Dieses unkonventionelle Auftreten entspricht der Selbsteinschätzung vieler junger Abgeordneter, die Reiser u.a. anhand einer aktuellen Interviewstudie zur parlamentarischen Sozialisation und Parlamentskultur in den Landtagen Baden-Württembergs und Nordrhein-Westphalens nachzeichnen. Auch wenn hier keine Bundestagsabgeordneten (die bei ihrem ersten Einzug in den Bundestag in der Regel mehr Parlamentserfahrung mitbringen dürften als Landtagsabgeordnete) interviewt wurden und nicht explizit nach dem Abstimmungsverhalten gefragt wurde, geht doch eine Mehrzahl der Neulinge davon aus, dass „sie ihre inhaltlichen Ziele und Vorhaben bereits im Laufe der ersten Wahlperiode anstoßen und realisieren können " (Reiser u.a., 2011, S. 829). Diese Befunde können zudem als Hinweis gelesen werden, dass der sogenannte „Fraktionszwang“ in der parlamentarischen Praxis tatsächlich geringer ausfällt als häufig angenommen wird (Patzelt, 1998). Oder, falls doch Fraktionszwang bestehen sollte, dass gerade junge Abgeordnete trotzdem nicht strategisch vorgehen, sondern gemäß ihrer Überzeugung abstimmen und eventuelle Konsequenzen in Kauf nehmen. Gleichzeitig muss an dieser Stelle aber noch einmal unterstrichen werden, dass auch in der Euro-Krise die ganz überwiegende Mehrheit der Abgeordneten aller Parteien im Einklang mit ihren Fraktionsspitzen abgestimmt hat - ob aus Fraktionszwang oder aus innerer Überzeugung (oder einer Mischung aus beidem) kann wohl nur jeder Abgeordnete für sich selbst beantworten.

\section{Fachliche Expertise}

Ein dritter Einflussfaktor, von dem abweichendes Stimmverhalten abhängen könnte, der aber in der Literatur noch keine Beachtung gefunden hat, ist die fachliche Expertise, konkret das Fachwissen der einzelnen Abgeordneten zu den Euro-Rettungsmaßnahmen, über die sie zu entscheiden haben. Fraktionen sind stark arbeitsteilig organisiert, weil sich aus Kapazitätsgründen nicht jeder Abgeordnete mit jeder zu entscheidenden Sachfrage detailliert auseinandersetzen kann, d.h. die Abgeordneten spezialisieren sich auf ausgewählte Politikbereiche (Schüttemeyer, 1998, S. 37 f.). Alle Fraktionen haben heute Arbeitsgruppen gebildet, in denen Experten der eigenen Partei zusammenkommen, bevor sie in den offiziellen Ausschüssen des Bundestages auf die Vertreter der anderen Fraktionen treffen (Ismayr, 2012, S. 127). Daraus kann die These abgeleitet werden, dass Abgeordnete, die sich in ihrer Arbeit nicht zuerst auf die Lösung der Euro-Krise konzentrieren, bei Abstimmungen möglicherweise verstärkt auf die Beschlussempfehlungen ihrer Fraktionsspitzen vertrauen. Gerade nach namentlichen Abstimmungen muss jeder Abgeordnete damit rechnen, aufgefordert zu werden, seine Entscheidung öffentlich zu rechtfertigen, was umso schwerer fallen dürfte, je weniger man sich mit der jeweiligen Thematik auskennt. Im Strom der Fraktionskollegen mitzuschwimmen, ist dann der wesentlich bequemere Weg. Dementsprechend wäre andererseits zu erwarten, dass Abgeordnete, die sich intensiv mit allen Facetten der Europapolitik und speziell der Euro-Krise beschäftigen und deswegen über die zur Abstimmung vorliegenden Gesetze (und deren ökonomische Folgen) besser informiert sind, stärker von der Fraktionslinie abweichen. Zudem ist Abgeordneten mit hoher Fachexpertise eher zuzutrauen, dass sie sich mit der Thematik der Euro-Krise gut genug auskennen, um ihr Abstimmungsverhalten auch vor der eigenen Fraktion verteidigen zu können. Dementsprechend geht das dritte Erklärungsmodell davon aus, dass abweichendes Stimmverhalten in der Euro-Krise mit der fachlichen Expertise der einzelnen Abgeordneten ansteigt.

Die fachliche Expertise der Abgeordneten wurde anhand der Mitgliedschaft in den Ausschüssen des Bundestages bestimmt. Je nachdem, auf welche Politikfelder sich Abgeordnete spezialisiert haben, wirken sie in Ausschüssen an der Formulierung von Beschlussempfehlungen für das Plenum mit. $\mathrm{Zu}$ Beginn der 17. Legislaturperiode sind 22 ständige Ausschüsse eingerichtet worden, die ein breites Spektrum an Politikbereichen abdecken. Diese 22 Ausschüsse wurden vom Autor danach unterschieden, ob ihre inhaltliche Arbeit eine thematische Nähe zur Euro-Krise aufweist oder nicht. Als 


\begin{tabular}{|l|c|c|c|c|c|c|c|c|}
\hline \multirow{2}{*}{ Fraktion } & \multicolumn{4}{|c|}{ Abgeordnete gesamt (620) } & \multicolumn{4}{c|}{ Abweichler (89) } \\
& \multicolumn{2}{|c|}{ Expertise } & \multicolumn{2}{c|}{ Keine Expertise } & \multicolumn{3}{c|}{ Expertise } & \multicolumn{2}{c|}{ Keine Expertise } \\
\cline { 2 - 10 } & $\mathrm{N}$ & $\%$ & $\mathrm{~N}$ & $\%$ & $\mathrm{~N}$ & $\%$ & $\mathrm{~N}$ & $\%$ \\
\hline CDU/CSU & 69 & 29,1 & 168 & 70,9 & 11 & 47,8 & 12 & 52,2 \\
\hline SPD & 43 & 29,5 & 103 & 70,6 & 11 & 31,4 & 24 & 68,6 \\
\hline FDP & 28 & 30,1 & 65 & 69,9 & 5 & 35,7 & 9 & 64,3 \\
\hline Die Linke & 21 & 27,6 & 55 & 72,4 & 0 & - & 0 & - \\
\hline Bgo/Grüne & 15 & 22,1 & 53 & 77,9 & 6 & 35,3 & 11 & 64,7 \\
\hline Gesamt & 176 & 28,4 & 444 & 71,6 & 33 & 37,1 & 56 & 62,9 \\
\hline
\end{tabular}

Quelle: Eigene Auszählung auf Basis DIP (Dokumentations- und Informationssystem für Parlamentarische Vorgänge) des Deutschen Bundestages (http://d ipbt.bundestag.de); endgültige Plenarprotokolle s. Tabelle 2; fachliche Expertise: Ordentliche Mitgliedschaft in Ausschüssen (http://www.bundestag.de/bu ndestag/ausschuesse17/index.jsp, Stand: 03/2013

Basis für die Zuordnung dienten die Kurzbeschreibungen der Ausschüsse auf der Homepage des Bundestages, in denen darüber informiert wird, welche Sachbereiche in die Zuständigkeit des jeweiligen Ausschusses fallen. Eine thematische Nähe zur Euro-Krise wurde unterstellt, wenn in der Beschreibung entweder auf EU-Wirtschaftspolitik, EU-Finanzpolitik oder allgemein Europapolitik ausdrücklich Bezug genommen wird. In diese Kategorie fallen folgende fünf Ausschüsse: (1) Ausschuss für die Angelegenheiten der Europäischen Union; (2) Haushaltsausschuss; (3) Ausschuss für Wirtschaft und Technologie; (4) Rechtsausschuss; (5) Finanzausschuss. Abgeordnete, die in mindestens einem dieser fünf Ausschüsse als ständiges Mitglied aktiv sind, sollten über eine höhere fachliche Expertise verfügen als Abgeordnete, die nicht in einem dieser Ausschüsse sitzen.

Tabelle 6 zeigt den Einfluss der fachlichen Expertise der einzelnen Abgeordneten auf ihr Abstimmungsverhalten in der Euro-Krise. In der linken Hälfte der Tabelle ist zunächst aufgeführt, wie sich alle 620 Abgeordneten der jeweiligen Fraktionen auf die Ausschüsse mit und ohne Expertise verteilen. Die Sitzverteilung in den Ausschüssen (Anzahl der Abgeordneten jeder Fraktion) wird entsprechend dem Kräfteverhältnis im Bundestag berechnet, sodass die Regierungsfraktionen in allen Ausschüssen knapp in der Mehrheit sind. Die Abgeordneten der fünf Fraktionen verteilen sich dabei je nach Ausschussgröße anteilig auf die einzelnen Ausschüsse. Demzufolge sitzen etwa 30 Prozent der Abgeordneten jeder Fraktion in den Ausschüssen mit fachlicher Nähe zur Euro-Krise (Expertise) und ca. 70 Prozent der Abgeordneten jeder Fraktion in Ausschüssen ohne thematische Nähe zur Euro-Krise (keine Expertise). ${ }^{9}$

Die rechte Seite der Tabelle zeigt, wie sich die Abweichler der Fraktionen auf die Ausschüsse mit und ohne thematische Nähe zur Euro-Krise verteilen. Diese Daten belegen, dass Abgeordnete mit fachlicher Expertise im Schnitt häufiger von der Fraktionslinie abgewichen sind als ihre Fraktionskollegen, die in fachfremden Ausschüssen sitzen. Besonders groß ist die Differenz in der CDU/CSU-Fraktion, denn hier sind die Abweichler mit Expertise (47,8 Prozent) im Vergleich mit allen CDU/CSU-Abgeordneten mit Expertise (29,1 Prozent) deutlich überrepräsentiert. Knapp die Hälfte der 23 CDU/ CSU-Abweichler saß in einem Ausschuss mit fachlicher Nähe zur Euro-Krise. Gerade diejenigen CDU/CSU-Abgeordneten, die sich in ihrer Parlamentstätigkeit intensiv mit Europapolitik und speziell der Euro-Krise befassen, haben die Euro-Rettungsmaßnahmen der Bundesregierung also signifikant häufiger abgelehnt. Dieser Zusammenhang zeigt sich besonders stark bei den fünf „Kettenabweichlern“ in der Fraktion, von denen vier über fachliche Expertise verfügen. Eine Tendenz in die gleiche Richtung lässt sich für FDP und Bündnis 90/Die Grünen feststellen, denn auch hier saßen mit 35,7 Prozent bzw. 35,3 Prozent überdurchschnittlich viele Abweichler in Ausschüssen mit thematischer Nähe zur Euro-Krise. Nur in der SPD-Fraktion ist der Zusammenhang verschwindend gering, d.h. hier hing abweichendes Stimmverhalten nur unwesentlich von der fachlichen Expertise der Abgeordneten ab. Gleiches gilt natürlich auch für die Fraktion der Linkspartei, in der ja keine Abgeordneten von der Parteilinie abgewichen sind, also auch nicht diejenigen mit fachlicher Expertise. Insgesamt jedoch waren 37,1 Prozent der Abweichler Mitglied in einem der Ausschüsse mit thematischer Nähe zu Euro-Krise, während nur 28,4 Prozent aller Abgeordneten fachliche Expertise zugeschrieben werden kann. Folglich kann das dritte Erklärungsmodell, nach dem Abgeordnete mit fachlicher Expertise in der Euro-Krise häufiger von der Fraktionslinie abweichen als ihre Fraktionskollegen ohne fachliche Expertise, bestätigt werden.

Dieses Ergebnis könnte man so interpretieren, dass die große Mehrheit der Abgeordneten ohne spezielle Fachkenntnisse sozusagen „blind“ auf ihre Fraktionsspitzen vertraut, während die Experten in den Fraktionen den auf europäischer Ebene getroffenen Vereinbarungen skeptischer gegenüberstehen, weil sie glauben, besser einschätzen zu können, ob damit die aktuellen Probleme adäquat zu lösen sind. Natürlich kann

9 Abgeordnete der Linkspartei und Bündnis 9o/Die Grünen sind in den Ausschüssen mit fachlicher Nähe zur Euro-Krise mit 27,6 Prozent bzw. 22,1 Prozent etwas unterrepräsentiert, was auf das komplizierte Berechnungsverfahren zur proportionalen Sitzverteilung in den Ausschüssen zurückzuführen ist; siehe dazu http://www.bundestag.de/ bundestag/ausschuesse17/azur/index.html (Abruf am 16.7.2013). 
nicht ausgeschlossen werden, dass gerade diejenigen Abgeordneten, die in Ausschüssen mit thematischer Nähe zur EuroKrise mitwirken, fraktionsübergreifend ähnliche wirtschaftspolitische Positionen vertreten und deswegen eher abweichen als ihre Parteikollegen. Dennoch gibt diese Diskrepanz einen ersten Hinweis darauf, dass viele fachfremde Abgeordnete den gestiegenen Anforderungen durch die neuen Mitwirkungsrechte des Bundestages in der Euro-Krise (noch) nicht gewachsen sind. Manche Parlamentarier gestehen sogar selbst unumwunden ein, dass ihre Expertise in komplexen Fragen der europäischen Finanz- und Wirtschaftspolitik immer noch unterentwickelt ist (Buzogány \& Kropp, 2013, S. 11). Zumindest bei Abstimmungen zu Euro-Rettungsmaßnahmen neigt die ganz überwiegende Mehrheit dieser Abgeordneten dann offensichtlich zu einer verstärkten Anpassung an die Fraktionslinie - ob sich dieses Muster auch in anderen Politikfeldern zeigt, kann im Kontext dieser Studie naturgemäß nicht geklärt werden.

\section{Zusammenfassung und Schlussfolgerungen}

Die politischen Maßnahmen zur Stabilisierung der EuroZone, die die Bundesregierung dem Bundestag zur Entscheidung vorgelegt hat, waren nicht nur zwischenparteilich, sondern auch innerparteilich sehr umstritten. Außer in der Linkspartei formierten sich in allen Bundestagsfraktionen kleine Gruppen von Abgeordneten, die die Beschlüsse zur Euro-Krise nicht mittragen wollten und bei namentlichen Abstimmungen von der fraktionellen Mehrheitsmeinung abgewichen sind.

In diesem Aufsatz wurden drei Erklärungsmodelle für abweichendes Abstimmungsverhalten in der Euro-Krise theoretisch hergeleitet und empirisch getestet: Das erste Modell ging davon aus, dass Abgeordnete, die mit einem Direktmandat in den Bundestag eingezogen sind, eher zu abweichendem Verhalten neigen als Abgeordnete mit Listenmandat, weil sie von ihrer Partei strukturell weniger abhängig sind. Diese These konnte eingeschränkt nur für die CDU/CSU-Fraktion bestätigt werden, während dieser Zusammenhang für die anderen Fraktionen nicht nachzuweisen war. Das zweite Modell vermutete einen Einfluss der Anzahl an Legislaturperioden auf das Abstimmungsverhalten der Abgeordneten. Auch hier ließ sich die These, dass abweichendes Stimmverhalten in der Euro-Krise mit parlamentarischer Erfahrung und Routine ansteigt, nicht bestätigen. Im Gegenteil: Besonders in den kleinen Parteien FDP und Bündnis 90/Die Grünen befanden sich numerisch die meisten Abweichler in der ersten Wahlperiode und in der CDU/CSU-Fraktion haben gerade die altgedienten Parlamentarier im Einklang mit der Mehrheit ihrer Kollegen gestimmt. Das dritte Modell ging davon aus, dass abweichendes Stimmverhalten in der Euro-Krise mit der fachlichen Expertise der einzelnen Abgeordneten ansteigt, weil sie eher in der Lage sind, ihre Gegenmeinung anschließend öffentlich und innerhalb der Partei zu verteidigen. Dieser Zusammenhang konnte für alle Fraktionen (außer der Linkspartei) mehr oder weniger stark nachgewiesen werden: Abgeordnete, denen eine hohe fachliche Expertise im Bereich der Europapolitik und speziell der Euro-Krise zugeschrieben werden kann, haben überdurchschnittlich häufig gegen die Euro-Rettungsmaßnahmen der Bundesregierung gestimmt. Dieses Ergebnis stützt die These, dass abweichendes Abstimmungsverhalten häufig weniger auf strukturelle Faktoren zurückzuführen ist, sondern mit persönlichen Merkmalen der Abgeordneten erklärt werden kann. So konnten schon Baumann et al. (2013) am Beispiel der namentlichen Abstimmung zur Neuregelung der Präimplantationsdiagnostik nachweisen, dass katholische Mitglieder des Bundestages fraktionsübergreifend den Gesetzentwurf zur eingeschränkten Zulassung der PID signifikant häufiger abgelehnt haben als evangelische und konfessionslose Abgeordnete. Bislang wurde jedoch in noch keiner Studie der Einfluss von fachlicher Expertise auf der Ebene des einzelnen Abgeordneten auf sein Abstimmungsverhalten nachgewiesen, sodass die vorliegenden Ergebnisse den Forschungsstand erweitern.

Die Bestätigung des dritten Modells deutet darüber hinaus auf ein Missverhältnis zwischen der parlamentarischen Kontrolle des Bundestages in der Euro-Krise und einer verantwortungsvollen Wahrnehmung dieser Kontrollfunktion hin. Der Bundestag bzw. seine Mitglieder haben sich in den letzten Jahren weitreichende formal-rechtliche Mitwirkungsrechte in der Europapolitik erstritten, insbesondere wenn durch Entscheidungen auf europäischer Ebene die Hoheit über das Haushaltsrecht berührt wird. Gemäß der jüngsten Rechtsprechung des Bundesverfassungsgerichts muss der Bundestag auch oder gerade in Zeiten der Euro-Krise die Budgethoheit behalten und allen Kreditvergaben durch den ESM gesondert zustimmen (Kube, 2012), sodass zukünftig regelrechte Abstimmungsmarathons zu immer detaillierteren finanzpolitischen Sachverhalten zu erwarten sind. Die Bundesregierung hat somit bei Verhandlungen in Brüssel stets den nationalen Gesetzgeber im Hinterkopf zu behalten, um die parlamentarische Ratifikation der vereinbarten Maßnahmen garantieren zu können. Auf der anderen Seite steht der Bundestag nun in der Pflicht, diese neue Aufgabe trotz der weiterhin bestehenden Vormachtstellung der Exekutive in der Europapolitik angemessen wahrzunehmen. Zukünftig können Abgeordnete bei politischen Fehlentwicklungen in der Wirtschaft- und Finanzpolitik der EU eben nicht mehr auf die Regierung verweisen, sondern werden selbst zu Adressaten von Kritik, weil sie als letzte Legislativinstanz aktiv zugestimmt haben. Der Nexus politischer Verantwortlichkeit hat sich damit in der Euro-Krise fundamental verschoben, weil sich die Mitglieder des Bundestages positionieren müssen, wenn die Regierung einen Gesetzentwurf vorlegt, und damit unabhängig von ihrer Entscheidung erhebliche Begründungslasten auf sich nehmen (Sinner, 2012).

Damit soll explizit nicht unterstellt werden, dass Abgeordnete mit einer hohen fachlichen Expertise zur Euro-Krise die bisherige Ausrichtung der Euro-Rettungspolitik aus guten Gründen bzw. berechtigterweise kritischer sehen. Ein gewisses $\mathrm{Ma}$ an Information und Wissen ist aber eine zwingende Voraussetzung, damit einfache Abgeordnete überhaupt in Erwägung ziehen können, gegen den Strom ihrer Fraktionsmehrheit 
anzuschwimmen. Ansonsten haben sie faktisch keine echte Wahloption, weil ihnen die finanz- und wirtschaftspolitischen Argumente fehlen, um ihre abweichende Meinung begründen zu können. Nach wie vor konzentrieren sich viele Abgeordnete zuerst auf ihre lokale Wahlkreisarbeit, in der Europapolitik bislang kaum von Bedeutung war. Die Einarbeitung in neue Wissensbereiche setzt jedoch einen mitunter langwierigen Lernprozess voraus, der nicht von heute auf morgen zu bewältigen ist (Buzogány \& Kropp, 2013). Aus diesem Blickwinkel kann die (vor-)schnelle Ausweitung parlamentarischer Mitwirkungsrechte in der Euro-Krise durchaus kritisch gesehen werden, weil sie den Bürgern eine Kontrolle der Regierung suggeriert, die von vielen Kontrolleuren eventuell noch gar nicht geleistet werden kann.

Umso dringlicher wäre deswegen die Frage zu beantworten, wie eine effektive Politikberatung in Zeiten der Euro-Krise ausgestaltet werden sollte, um möglichst alle Abgeordneten in die Lage zu versetzen, die ihnen zugewiesenen Aufgaben mit
Überzeugung wahrnehmen zu können. Die zentrale Herausforderung besteht in einer frühzeitigen und kompakten Informationsübermittlung, welche Maßnahmen mit den zu beschließenden Gesetzentwürfen konkret umgesetzt werden sollen und vor allem welche ökonomischen Konsequenzen zu erwarten sind. Die Vorlagen der Bundesregierung waren jedoch bislang in einem für Nichtexperten nur schwer verständlichen technischen Sprachstil gehalten, zudem haben viele Abgeordnete beklagt, dass sie viel zu spät über die konkrete Umsetzung der Rettungsmaßnahmen informiert wurden, um diese im Detail beurteilen zu können. An dieser Stelle sollte eine Politikberatung durch unabhängige Wirtschaftsforschungsinstitute und Sachverständige ansetzen, die den Abgeordneten aller Fraktionen im Rahmen von Informationsveranstaltungen verschiedene Szenarien eröffnet, unter welchen Bedingungen die auf europäischer Ebene vorbereiteten Maßnahmen welche ökonomischen (und politischen) Folgewirkungen in der Eurozone nach sich ziehen könnten.

Anhang 1: Gesetze und Beschlüsse des Bundestages im Zusammenhang mit der Euro-Krise mit namentlicher Abstimmung (2010-2012)

\begin{tabular}{|c|c|c|c|}
\hline & Datum & Sitzung & Gesetz/Zustimmender Beschluss des Bundestages \\
\hline 1 & 7.5 .2010 & $17 / 41$ & $\begin{array}{l}\text { Gesetz zur Übernahme von Gewährleistungen zum Erhalt der für die Finanzstabilität in der Währungsunion } \\
\text { erforderlichen Zahlungsfähigkeit der Hellenischen Republik (Währungsunion-Finanzstabilitätsgesetz-WFStG) }\end{array}$ \\
\hline 2 & 21.5 .2010 & $17 / 44$ & $\begin{array}{l}\text { Gesetz zur Übernahme von Gewährleistungen im Rahmen eines europäischen Stabilisierungsmechanismus } \\
\text { (Stabilisierungsmechanismusgesetz - StabMechG) }\end{array}$ \\
\hline 3 & 29.9 .2011 & $17 / 130$ & $\begin{array}{l}\text { Gesetz zur Änderung des Gesetzes zur Übernahme von Gewährleistungen im Rahmen eines europäischen } \\
\text { Stabilisierungsmechanismus (StabMechGÄndG) }\end{array}$ \\
\hline 4 & 26.10 .2011 & $17 / 135$ & $\begin{array}{l}\text { Entschließungsantrag der Fraktionen CDU/CSU, SPD, FDP und BÜNDNIS 90/DIE GRÜNEN zu der Abgabe einer } \\
\text { Regierungserklärung durch die Bundeskanzlerin zum Europäischen Rat und zum Eurogipfel am 26. Oktober } \\
2011 \text { in Brüssel }\end{array}$ \\
\hline 5 & 27.2 .2012 & $17 / 160$ & $\begin{array}{l}\text { Einholung eines zustimmenden Beschlusses des Deutschen Bundestages nach § } 3 \text { Absatz } 1 \text { des Stabilisierungs- } \\
\text { mechanismusgesetzes (Stab-MechG) für Notmaßnahmen der Europäischen Finanzstabilisierungsfazilität } \\
\text { zugunsten der Hellenischen Republik („,Finanzhilfe Griechenland“) }\end{array}$ \\
\hline 6 & 29.6 .2012 & $17 / 188$ & $\begin{array}{l}\text { Gesetz zu dem Vertrag vom 2. März } 2012 \text { über Stabilität, Koordinierung und Steuerung in der Wirtschafts- und } \\
\text { Währungsunion („Fiskalpakt“) }\end{array}$ \\
\hline 7 & 29.6 .2012 & $17 / 188$ & $\begin{array}{l}\text { Gesetz zu dem Vertrag vom 2. Februar } 2012 \text { zur Einrichtung des Europäischen Stabilitätsmechanismus („ESM- } \\
\text { Gesetz“) }\end{array}$ \\
\hline 8 & 29.6 .2012 & $17 / 188$ & $\begin{array}{l}\text { Gesetz zur finanziellen Beteiligung am Europäischen Stabilitätsmechanismus (ESM-Finanzierungsgesetz - } \\
\text { ESMFinG) }\end{array}$ \\
\hline 9 & 29.6 .2012 & $17 / 188$ & $\begin{array}{l}\text { Gesetz zu dem Beschluss des Europäischen Rates vom 25. März } 2011 \text { zur Änderung des Artikels } 136 \text { des Vertrags } \\
\text { über die Arbeitsweise der Europäischen Union hinsichtlich eines Stabilitätsmechanismus für die Mitgliedstaa- } \\
\text { ten, deren Währung der Euro ist }\end{array}$ \\
\hline 10 & 19.7 .2012 & $17 / 189$ & $\begin{array}{l}\text { Einholung eines zustimmenden Beschlusses des Deutschen Bundestages nach § } 3 \text { Absatz } 1 \text { i. V. m. § } 3 \text { Absatz } 2 \\
\text { Nummer } 1 \text { und } 4 \text { des Stabilisierungsmechanismusgesetzes (Stab-MechG) für Notmaßnahmen der Europäi- } \\
\text { schen Finanzstabilisierungsfazilität zugunsten Spaniens („Finanzhilfe Spanien“) }\end{array}$ \\
\hline 11 & 30.11 .2012 & $17 / 212$ & $\begin{array}{l}\text { Einholung eines zustimmenden Beschlusses des Deutschen Bundestages nach §3 Absatz } 1 \text { i. V. m. §3 Absatz } 2 \\
\text { Nummer } 2 \text { des Stabilisierungsmechanismusgesetzes (StabMechG) („Änderungen im bestehenden Anpas- } \\
\text { sungsprogramm für Griechenland“) }\end{array}$ \\
\hline
\end{tabular}




\section{Literaturverzeichnis}

Baumann, M., Debus, M. \& Müller, J. (2013). Das legislative Verhalten von Bundestagsabgeordneten zwischen persönlichen Charakteristika, Wahlkreisinteressen und Parteilinie. Eine Untersuchung am Beispiel der Auseinandersetzung um die Präimplantationsdiagnostik. Zeitschrift für Politikwissenschaft, 23(2), 177-211.

Belke, A., \& Dreger, C. (2011). Das zweite Rettungspaket für Griechenland und Perspektiven für die Europäische Zentralbank. integration Vierteljahresschrift des Instituts für Europäische Politik, 03/2011, 214-227.

Buzogány, A., \& Kropp, S. (2013). Sisyphos und der Marmorblock: Parlament und Expertise in Zeiten der Euro-Krise. Zeitschrift für Politikberatung, 6(1), 3-14.

Buzogány, A., \& Kropp, S. (2013). Der Deutsche Bundestag im „Tal der Ahnungslosen"? Wissen und Expertise des Parlaments in europäisierten Fachpolitiken. In S. Kropp \& S. Kuhlmann (Hrsg.), Wissen und Expertise in Politik und Verwaltung, der moderne staat (dms), Sonderheft 01/2013, Opladen: Budrich,161-182.

Calliess, C., \& Schoenfleisch, C. (2012). Auf dem Weg in die europäische „Fiskalunion“? - Europa- und verfassungsrechtliche Fragen einer Reform der Wirtschafts- und Währungsunion im Kontext des Fiskalvertrages. JuristenZeitung (JZ), 67(10), 477-487.

Dittberner, J. (2003). Freies Mandat und politische Geschlossenheit: Widerspruch oder Ergänzung zweier Prinzipien des Parlamentarismus? Zeitschrift für Parlamentsfragen, 34(3), 550-564.

Fritzsche, E. (2009). Innerfraktionelle Geschlossenheit im Spiegel der Politikwissenschaft. Stand der Forschung und notwendige Weiterführungen. Zeitschrift für Parlamentsfragen, 40(3), 661-682.

Hölscheidt, S. (2013). Parlamentarische Kontrolle in der Eurokrise. In B. Eberbach-Born, A. Stuchlik, S. Kropp \& W. Zeh (Hrsg.), Parlamentarische Kontrolle und Europäische Union (S. 105-129). Baden-Baden: Nomos.

Holzhacker, R. (2005). The Power of Opposition Parliamentary Party Groups in European Scrutiny. Journal of Legislative Studies, 11(3/4), 428-445

Hufeld, U. (2011). Zwischen Notrettung und Rütlischwur: der Umbau der Wirtschafts- und Währungsunion in der Krise. integration - Vierteljahresschrift des Instituts für Europäische Politik, 02/2011, 117-131.

Isensee, J. (2007). Fraktionsdisziplin und Amtsgewissen: Verfassungsrechtliche Garantie der Freiheit des Mandats im politischen Prozess. In W. Patzelt, M. Sebaldt \& U. Kranenpohl (Hrsg.), Res publica semper reformanda: Wissenschaft und politische Bildung im Dienste des Gemeinwohls, Festschrift für Heinrich Oberreuter zum 65. Geburtstag (S. 254-267), Wiesbaden: VS Verl. für Sozialwissenschaften.

Ismayr, W. (2012). Der Deutsche Bundestag, 3. völlig überarb. und aktualisierte Aufl., Wiesbaden: Springer VS.

Klein, H. H. (2012). Die Macht des Bundestages. Zeitschrift für Gesetzgebung, 27(3), 209-227.

Kube, H. (2012). Nationale Budgethoheit und Europäische Integration. Archiv des öffentlichen Rechts, 137(2), 205-222.

Kunstein, T., \& Wessels, W. (2011). Die Europäische Union in der Währungskrise: Eckdaten und Schlüsselentscheidungen. integration - Vierteljahresschrift des Instituts für Europäische Politik, 04/2011, 308-322.

Manow, P. (2012). Wahlkreis- oder Listenabgeordneter, Typus oder Episode? Eine Sequenzanalyse der Wege in den Bundestag. Politische Vierteljahresschrift, 53(1), 53-78.

Manow, P., \& Nistor, M. (2009). Wann ist ein Listenplatz sicher? Eine Untersuchung der Bundestagswahlen 1953 bis 2002. Zeitschrift für Parlamentsfragen, 40(3), 603-620.

Müller-Graff, P.-Ch. (2011). Euroraum-Budgethilfenpolitik im rechtlichen Neuland, integration - Vierteljahresschrift des Instituts für Europäische Politik, 04/2011, 289-307.

Ondarza, N. v. (2013): Auf dem Weg zur Union in der Union. Institutionelle Auswirkungen der differenzierten Integration in der Eurozone auf die EU. integration - Vierteljahresschrift des Instituts für Europäische Politik, 01/2013, 17-33.

Patzelt, W. J. (1998). Wider das Gerede vom „Fraktionszwang“! Funktionslogische Zusammenhänge, populäre Vermutungen und die Sicht der Abgeordneten. Zeitschrift für Parlamentsfragen, 29(2), 323-347.

Patzelt, W. J. (1999). What Can an Individual MP Do in German Parliamentary Politics? Journal of Legislative Studies, 5(3-4), 23-52.

Patzelt, W. J. (2003). Party Cohesion and Party Discipline in German Parliaments. Journal of Legislative Studies, 9(4), 102-115.

Reiser, M., Hülsken, C., Schwarz, B., \& Borchert, J. (2011). Das Reden der Neulinge und andere Sünden. Parlamentarische Sozialisation und Parlamentskultur in zwei deutschen Landtagen. Zeitschrift für Parlamentsfragen, 42(4), 820-834.

Saalfeld, T. (1995). Parteisoldaten und Rebellen: Eine Untersuchung zur Geschlossenheit der Fraktionen im Deutschen Bundestag (1949-1990). Opladen: Leske \& Budrich.

Saalfeld, T. (2005). Determinanten der Fraktionsdisziplin: Deutschland im internationalen Vergleich. In S. Ganghof \& P. Manow (Hrsg.), Mechanismen der Politik - Strategische Interaktion im deutschen Regierungssystem (S. 35-71). Frankfurt am Main: Campus.

Sarcinelli, U. (1989). Parlamentarische Sozialisation in der Bundesrepublik Deutschland zwischen politischer „Sonderkultur“ und Basislegitimation, Zeitschrift für Parlamentsfragen, 20(3), 388-407.

Schüttemeyer, S. S. (1998). Fraktionen im Deutschen Bundestag: Empirische Befunde und theoretische Folgerungen, Opladen: Westdeutscher Verlag.

Schüttemeyer, S. S. (2002). Wer wählt wen wie aus? Pfade in das unerschlossene Terrain der Kandidatenaufstellung, Gesellschaft - Wirtschaft - Politik, 51(2), 145-161.

Schüttemeyer, S. S., \& Sturm, R. (2005). Der Kandidat - das (fast) unbekannte Wesen. Befunde und Überlegungen zur Aufstellung der Bewerber zum Deutschen Bundestag, Zeitschrift für Parlamentsfragen 36(3), 539-553.

Seidel, M. (2010). Aktuelle Probleme der europäischen Währungsunion. integration - Vierteljahresschrift des Instituts für Europäische Politik, 04/2010, 334-349.

Sieberer, U. (2006). Party Unity in Parliamentary Democracies: A Comparative Analysis, Journal of Legislative Studies, 12(2), 150-178.

Sieberer, U. (2010). Behavioral Consequences of Mixed Electoral Systems: Deviating Voting Behavior of District and List MPs in the German Bundestag. Electoral Studies, 29, 484-496.

Sinner, S. (2012). Der Deutsche Bundestag als zentrales Verfassungsorgan nach der neuen Rechtsprechung des Bundesverfassungsgerichts. Zeitschrift für Parlamentsfragen, 43(2), 313-323.

Stecker, C. (2011). Bedingungsfaktoren der Fraktionsgeschlossenheit. Eine vergleichende Analyse der deutschen Länderparlamente. Politische Vierteljahresschrift, 52(3), 424-447.

Wimmel, A. (2012). Deutsche Parteien in der Euro-Krise: Das Ende des Konsensprinzips? integration - Vierteljahresschrift des Instituts für Europäische Politik, 01/2012, 19-34.

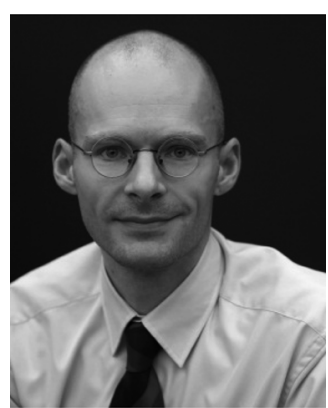

Dr. Andreas Wimmel vertritt die Professur „Politisches System Deutschlands und der EU“ an der Universität Oldenburg und ist Universitätsassistent am Institut für Politikwissenschaft der Universität Innsbruck (zurzeit beurlaubt). Email: andreas.wimmel@uibk.ac.at 